\title{
Saving Energy in Powerwash Systems
}

\begin{abstract}
A new spray cleaner for the surface cleaning of plastic parts achieves the required result already at room temperature and thus considerably reduces the energy consumption in Powerwash systems. The focus is on energy efficiency and environmental compatibility.
\end{abstract}

Before plastic parts such as bumpers go into the paint shop, the workpieces must be cleaned and pre-treated. The cleanliness of the part surface is decisive for an optimum result in the coating process. In Powerwash systems, the plastic parts, for example bumpers, are treated with an aqueous cleaning solution at temperatures of usually 45 to $70^{\circ} \mathrm{C}$ by means of spray application. The high temperatures are necessary to dissolve stubborn dirt, but also to keep the baths low-foaming, thus preventing over-foaming and unnecessary maintenance. Modern painting systems usually run 24 hours 7 days a week in continuous operation. The process temperature must therefore also remain permanently high during pre-treatment. However, this causes enormous energy costs.

\section{Optimum results at low temperatures}

With the new Divinol 1362 KC spray cleaner from Zeller + Gmelin, considerable savings can be made in energy consumption, as the process-stable cleaner achieves the required surface cleanliness already at reduced bath temperatures of $25^{\circ} \mathrm{C}$.

The necessary heating up and the continuous maintenance of high process temperatures are eliminated. The pre-treatment and thus the entire painting process become more economical.

A low pre-treatment temperature provides numerous advantages.

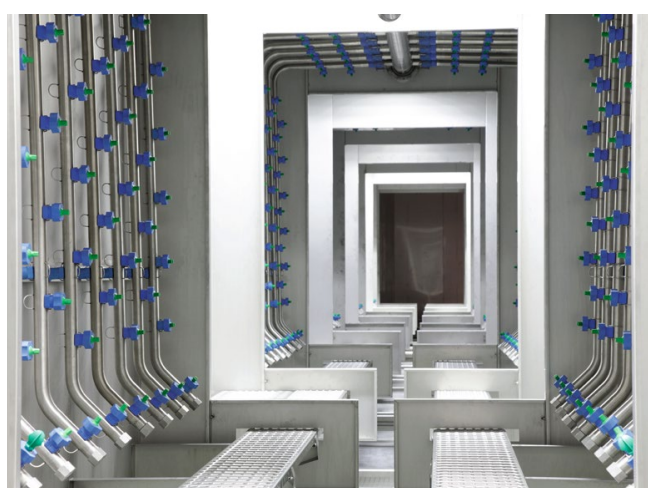

In Powerwash systems, plastic parts are treated with an aqueous cleaning solution at temperatures of usually 45 to $70^{\circ} \mathrm{C}$ by means of spray application.

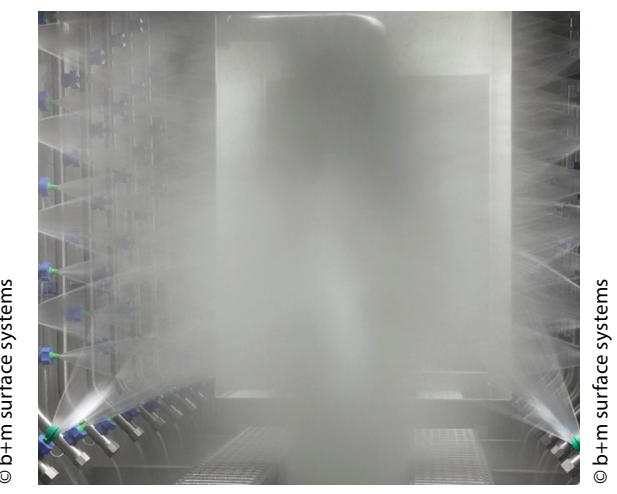

The new spray cleaner significantly reduces the energy required for Powerwash systems, as it already achieves the required cleaning performance at room temperature.
Various alkaline and acidic cleaners are available for a wide range of applications - some of which are free of labelling. The spray cleaner is suitable for all common plastics, has a high biostability used without disturbing foam formation. Due to the low process temperature, less water evaporates during operation of the Powerwash system, which also results in savings in fresh water consumption.

The topic of sustainability and climate protection is very important to the longestablished company from Eislingen. In January 2020, Zeller + Gmelin qualified as a "climate neutral location". All products - including the new spray cleaner - are produced, stored and delivered in a climate-neutral manner. //

\section{Contact}

Zeller+Gmelin GmbH \& Co. KG

Eislingen/Fils (Germany)

info@zeller-gmelin.de

www.zeller-gmelin.de 